\title{
The Instability Strip of M3
}

\author{
G. Á. Bakos, J. Jurcsik \\ Konkoly Observatory, P.O. Box 67, H-1525 Budapest XII, Hungary
}

\begin{abstract}
We present new multicolour CCD photometry of the central part of the globular cluster M3, mapping the precise position of $~ 120$ RR Lyrae stars (RRab, RRd, RRc) on the horizontal branch (HB). The location of the double-mode variables (RRd) is in perfect agreement with recent theoretical results. We find a significant internal spread of metallicity amongst the RRab variables.
\end{abstract}

CCD observations were carried out using the 1-m RCC telescope at the Piszkéstetö Mountain Station of Konkoly Observatory. Standard crowded field photometry with ALLFRAME (Stetson, 1994) yielded light curves with complete phase coverage for $\sim 120$ RR Lyrae stars, as well as precise magnitudes for the nonvariable stars of the HB. A detailed discussion of observations and data reduction will be given in a forthcoming paper (Bakos et al. 1999).

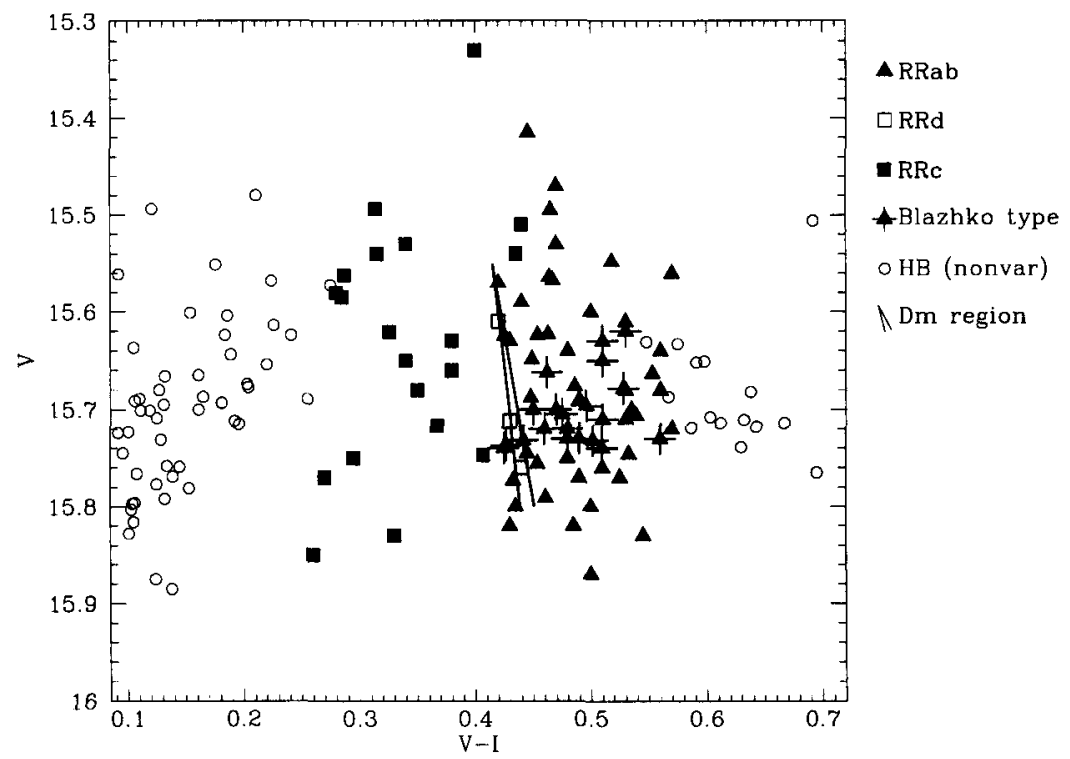

Figure 1. The horizontal branch of M3 with the observed RR Lyrae stars plotted. The double-mode regime (Szabó et al. 2000) is also shown. 
The colour-magnitude diagram of the $\mathrm{HB}$ was constructed using the weighted average magnitude of the best $\sim 15$ frames for the non-variable stars, while the magnitudes and colours for all known variables were derived from optimal-order Fourier fits to the light curves. Fig. 1 reveals that the HB has a considerable width, notably exceeding the photometric errors (which are estimated to be less than $0.03 \mathrm{mag}$ ). This can either be interpreted as an unusual stellar evolution scenario of the $\mathrm{HB}$, or a significant internal spread in the metallicity.

Blazhko variables have markedly smaller scatter in $V$; however, this might be due to small number statistics. The RRab and RRc populations are wellseparated, with minimal overlap. The RRd regime is relatively narrow in colour, though it should be noted that only three of the five known RRd variables are within our observed fields. The position of the RRd stars is in perfect agreement with the theoretical prediction of Szabó et al. (2000) using doublemode RR Lyrae models with $Z=0.001$, and $M_{R R}=0.65 M_{\odot}$. Bolometric corrections and temperature-colour transformations were derived by employing adequate grids of stellar atmosphere models from Castelli, Gratton, \& Kurucz (1997). The observations precisely mapped the morphology of various parts of the HB, which can serve as a check on further theoretical calculations.

Metallicity and reddening of the single-mode RRab stars were determined by applying relations between their physical and light curve parameters (Jurcsik \& Kovács 1996, Kovács \& Jurcsik 1997). The average metallicity of the cluster turns out to be $[\mathrm{Fe} / \mathrm{H}]=-1.37 \pm 0.17$ (std. dev.), where the large scatter can neither be ascribed to photometric errors, nor to the inaccuracy in the aforementioned methods. The almost negligible reddening value of $E(V-I)=$ $0.00 \pm 0.02$ obtained from comparison of the observed and calculated colours agrees well with previous estimates.

Acknowledgments. This work was supported in part by OTKA T-024022 and T-030954.

\section{References}

Bakos, G. Á., et al. 1999, in preparation

Castelli F., Gratton, R. G., \& Kurucz, R. L. 1997, A\&A, 318, 841

Jurcsik, J. \& Kovács G. 1996, A\&A, 312, 111

Kovács G. \& Jurcsik, J. 1997, A\&A, 322, 218

Stetson, P. B. 1994, PASP, 106, 250

Szabó, R., Csubry, Z., Kolláth, Z., \& Buchler, R. 2000, in these proceedings, p. 374 